\title{
Kandidaturen für den Bundesvorstand
}

\section{- Präsidium und Kommissionsvorsitz -}

Die bisherigen Mitglieder des Präsidums haben signalisiert, dass sie erneut kandidieren wollen: Jutta Wagner für das Amt der Präsidentin, Renate Maltry und Ramona Pisal für die beiden Ämter der Vizepräsidentin und Dagmar Brinkmann für das Amt der Schatzmeisterin. Ihre Kandidatur als Vorsitzende einer Ständigen Kommission haben angekündigt:

\section{Kommission Arbeits-, Gleichstellungs- und Wirtschaftsrecht}

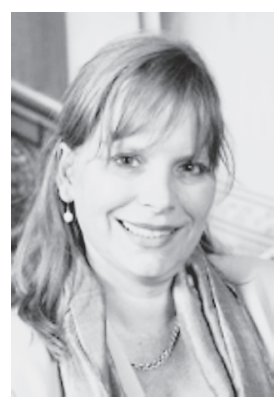

Prof. Dr. Marlene Schmidt, Rechtsanwältin, apl. Professorin für Bürgerliches Recht, Arbeitsrecht, Umweltrecht, Europarecht und Rechtsvergleichung an der Goethe-Universität Frankfurt am Main, geb. 1965, djb-Mitglied seit 1991, 1995 2001 Mitglied der Europa-Kommission des djb, von 2001-2005 und seit 2009 wieder Mitglied der Kommission Arbeits-, Gleichstellungs- und Wirtschaftsrecht, Diss. 1994 im europäischen Arbeitsrecht, Habil. 2006 im europäischen Privatrecht, über 70 Veröffentlichungen im Arbeitsrecht, davon viele zu Gleichstellungsfragen, bis Februar hauptberuflich Wissenschaftlerin, seit März 2008 Rechtsanwältin in Frankfurt am Main und ausschließlich im Arbeitsrecht tätig.

Auf der Liste der Themen, die ich in den nächsten vier Jahren mit der Kommission gerne vorantreiben möchte, steht ganz oben die Durchsetzung des Grundsatzes der Entgeltgleichheit von Frau und Mann, insbesondere für gleichwertige Arbeit. Ferner möchte ich künftig - in Zusammenarbeit mit der Kommission Öffentliches Recht, Europa- und Völkerrecht - dem öffentlichen Dienst einschließlich der Beamten und Richter und insbesondere dem auch dort zu beobachtenden Phänomen „gläserner Decken“, die die Beförderung von Beamtinnen und Richterinnen verhindern, mehr Aufmerksamkeit widmen. Weitere wichtige Themen sind die Wahl von Frauen in den Aufsichtsrat von Unternehmen, wo sie nach wie vor völlig unterrepräsentiert sind, sowie die kritische Begleitung der Arbeit der Anti-Diskriminierungsstelle des Bundes.

\section{Kommission Zivil-, Familien- und Erbrecht, Recht anderer Le- bensgemeinschaften}

Dr. Angelika Nake, Fachanwältin Familienrecht. Geboren am 6. September 1961 in Bad Hersfeld, Studium in Gießen, Referendariat in Köln, Promotionsstudium in Madrid. Promotionsthema: Der spanische gesetzliche Güterstand der Errungenschaftsgemeinschaft mit vergleichenden Ausführungen zum deutschen Recht. Getrennt lebend, zwei Kinder.

Begonnen habe ich als Rechtsanwältin in München, danach war ich in Düsseldorf, Darmstadt, Memmingen und nun wieder in Darmstadt tätig. Neben dem Schwerpunkt Familienrecht habe ich als die Kinder noch klein waren, rechtlich

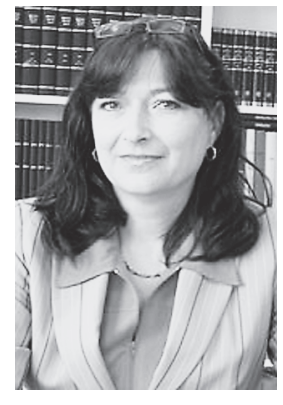

schwierige Betreuungen geführt. Was den Blick in die Wirtschaft schärft, da ich diverse Geschäfte geführt und aufgelöst habe, Vermögensanlagen betrieben und Grundstücke verkauft habe. Neben diesen Tätigkeiten hatte ich die verschiedensten Lehrtätigkeiten inne, beginnend mit der Ausbildung für Altenpflegerinnen über die Ausbildung von Verwaltungsfachangestellten und Dozententätigkeiten an den Fachhochschulen Aschaffenburg und Kempten.

An Veröffentlichungen habe ich neben Artikeln und Aufsätzen über verschiedenste Themen des Familienrechts an einer Monographie zum Thema „Gewalt im Alter“ im Bereich Aspekte der Geschäftsfähigkeit bei der rechtlichen Betreuung mitgewirkt und den Online-Kommentar Lexis-Nexis im Betreuungsrecht erstellt und betreut. Leider habe ich viel zu wenig Zeit, hier so tätig zu sein, wie es mir Spaß machen würde.

Im Juristinnenbund war ich fünf Jahre Mitglied der Kommission Europa, sieben Jahre Delegierte in der „National Coalition zur Durchsetzung der UN-Kinderrechtekonvention " und bin seit September 2005 die Vorsitzende der Kommission langen Namens, für die ich mich wieder bewerbe.

In den letzten vier Jahren ist im Familienrecht, wie man so schön sagt, kein Stein auf dem anderen geblieben. Wir haben an den Rechtänderungen zum Familienverfahrensrecht mitgewirkt, zum Versorgungsausgleich, zum Güterrecht, zum Erbrecht, zum Unterhaltsrecht und zum Vaterschaftsklärungsverfahren. Daneben haben wir die Position unseres Verbandes im europäischen Kontext eingebracht, zu dem geplanten deutsch/ französischen Wahlgüterstand und dem Internationalen Familienverfahrensrecht, um nur wenige zu nennen.

Diese neuen Gesetze werden in der nächsten Zeit angewandt und so sehe ich meine Aufgabe hier in der Begleitung der Umsetzung. Besonders in den Blick nehmen müssen wir hier das Unterhaltsrecht und das neue Familienverfahrensgesetz.

\section{Kommission Strafrecht}

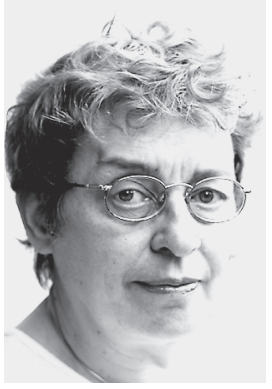

Dagmar Freudenberg, geb. 1952 in Emmern (jetzt: Emmerthal bei Hameln), Studium in Göttingen, verheiratet, zwei Kinder, seit 1978 in der Justiz in Niedersachsen, Staatsanwältin seit 1980 , seit 1981 Bearbeitung von Jugendstrafsachen. Von 1985 bis 1995 Lehrauftrag "Jugendrecht" am Erziehungswissenschaftlichen Fachbereich der Universität Göttingen. Von 1993 bis 31. März 2009 Bearbeitung des Sonderdezernats „Sexualstraftaten“, parallel dazu seit 2003 auch 
Bearbeitung des Sonderdezernats „Häusliche Gewalt“ bei der Staatsanwaltschaft Göttingen.

Seit 1994 Mitglied der Strafrechtskommission des djb, seit Ende 2001 Vorsitzende der djb-Kommission „Gewalt gegen Frauen und Kinder“, dazu seit 2001 für den djb Mitglied der Bund-Länder-AG „Häusliche Gewalt“ des BMFSFJ und seit 2003 Mitglied der Bund-Länder-AG „Schutz von Kindern und Jugendlichen vor sexueller Gewalt und Ausbeutung “ des BMFSFJ. Seit 2003 auf Bitte des Nds. Ministeriums der Justiz Mitarbeit als Ansprechpartnerin häusliche Gewalt aus der Staatsanwaltschaft im Praxisbeirat der Landeskoordinierungsstelle häusliche Gewalt beim Landespräventionsrat Niedersachsen und seit 1. April 2009 zur Hälfte der Arbeitskraft durch Abordnung tätig als Koordinatorin im Projekt häusliche Gewalt im Landespräventionsrat Niedersachsen, gleichzeitig Referentin für häusliche Gewalt und Stalking. 2006 und 2007 Mitglied in der AG 4 (UAG 1) des Bundesjustizministeriums zur Erarbeitung eines Nationalen Integrationsplans der Bundesregierung und schließlich seit 2002 auf Bitte des Nds. Ministeriums der Justiz Mitarbeit in der Stiftung Opferhilfe im Vorstand des Regionalen Opferhilfefonds Göttingen. Zahlreiche Vorträge, Stellungnahmen und Tagungsveröffentlichungen.

Die Fragen der Gleichberechtigung - und insbesondere des Schutzes der zumeist weiblichen Opfer von Gewaltstraftaten - sind mir seit Eintritt in mein Berufsleben, aber auch in der Arbeit des djb besonders wichtig. Ziel meiner Arbeit war und ist die Verwirklichung einer gerechten, gewaltfreien Gesellschaft. Hierfür bedarf es auch und gerade des Engagements in der Strafrechtskommission insbesondere für die Belange der schwachen kindlichen und zumeist weiblichen Opfer. Dazu gehört eine Stärkung der Rechte der Verletzten im Verfahren, zum Beispiel auf (psychosoziale) Opferbegleitung, ebenso wie die Wahrnehmung der Opferrechte im Rahmen des neu gesetzlich verankerten Deals oder - perspektivisch - eines Ausgleichs im weitesten Sinne (Restorative Justice). Hieran möchte ich ebenso arbeiten wie an der Verwirklichung der gleichberechtigten Gesellschaft und dafür die Möglichkeiten des Strafrechts und - insbesondere - des Strafprozessrechts nutzen und verbessern.

\section{Kommission Recht der sozialen Sicherung, Familienlasten- ausgleich}

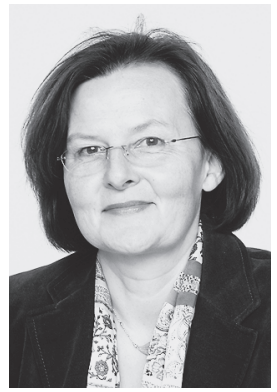

Prof. Dr. Margarete Schuler-Harms, geb. 1959, Studium in Freiburg, Professorin für Öffentliches Recht, insbes. Öffentliches Wirtschafts- und Umweltrecht an der Helmut-Schmidt-Universität/Universität der Bundeswehr in Hamburg, verheiratet, zwei Kinder. Mitglied im djb seit 1989, in der Kommission „Recht der sozialen Sicherung, Familienlastenausgleich“ seit 1997. Zahlreiche Veröffentlichungen zum Öffentlichen Familienrecht sowie zu Fragen der Gleichstellung im Zuwanderungs- und Flüchtlingsrecht. 2008 bis 2010 Mit- glied der Sachverständigenkommission zur Erstellung des Gleichstellungsberichts der Bundesregierung.

Die globalen Herausforderungen an die Wirtschaft und der demografische Wandel führen zu massiven Veränderungen der sozialen Sicherungssysteme, die die Kommission kritisch und mitunter auch mit eigenen konzeptionellen Vorschlägen begleitet. Für die Alterssicherung sind weitere Strukturreformen zu erwarten. Ein geschlechtergerechtes djb-Rentenmodell liegt hier bereits seit 2001 vor. Vor besonderen Herausforderungen steht auch die vorwiegend von Frauen geleistete familiäre und erwerbsmäßige Pflege. Dienstleistungsverhältnisse im privaten und sozialen Sektor und ihre sozialrechtliche Absicherung werden deshalb einen besonderen Schwerpunkt der Kommissionsarbeit in den kommenden Jahren bilden.

Die Kommission wird weiterhin konstruktiv und kritisch die Bemühungen der Politik um eine verbesserte, an der Vereinbarkeit von Beruf und Familie orientierte Familienpolitik begleiten. Die Leistungen nach „Hartz IV“ und ihre geschlechterpolitischen Implikationen beschäftigen die Kommission bereits seit längerem intensiv. Im Lichte der aktuellen Wirtschaftskrise erhalten darüber hinaus latente Themen wie die steuerlichen Be- und Entlastungen von Familien, Kindergeld, Kinderzuschlag und Forderungen nach einem Kindesgrundeinkommen neue Aktualität.

\section{Kommission Öffentliches Recht, Europa- und Völkerrecht}

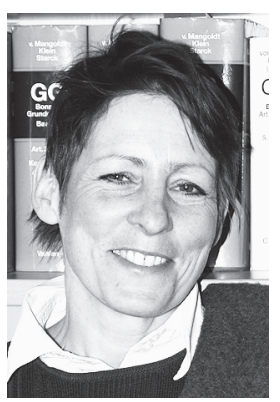

Dr. Katja Rodi, geb. 1959 in Göttingen, verheiratet, zwei Kinder, Studium in Göttingen und München, Schwerpunkt Völker- und Europarecht, Anwältin von 1988-1994 in München, 1994-1999 wiss. Mitarbeiterin in Rostock, Promotion mit naturschutz- und finanzverfassungsrechtlicher Thematik, seit 1999 wiss. Mitarbeiterin für bes. Lehraufgaben und Fakultätsgleichstellungsbeauftragte in Greifswald. Vorstandsmitglied des Landesfrauenrates Mecklenburg-Vorpommern. Mitglied im djb seit 1986, 2001 bis 2003 Mitglied des Arbeitsstabes Juristinnenausbildung, 2002 Gründung der Regionalgruppe Vorpommern des djb und Regionalgruppenvorsitzende, seit 2005 Vorsitzende der Kommission Öffentliches Recht, Europa- und Völkerrecht.

Programm für die zukünftige Kommissionsarbeit: Allgemein: Beobachtung und Begleitung von Gesetzgebungsvorhaben in den Arbeitsbereichen der Kommission einschließlich aller das Migrationsrecht betreffender Fragen durch Stellungnahmen, Pressemitteilungen, Teilnahme an Anhörungen etc.; Vorbereitung des nächsten Bundeskongresses in Zusammenarbeit mit der Kommission Arbeits-, Gleichstellungs- und Wirtschaftsrecht. Völkerrecht: Weitere Begleitung des Follow-upProzesses von der UN-Frauenrechtskonvention (CEDAW), insbesondere Schattenbericht zum Zwischenbericht der Bundesregierung. Europarecht: Neue Gender Equality Roadmap der Kommission, insbesondere Geschlechtergleichstellung bei Bildung und sozialen Vergünstigungen im ausstehenden Anti- 
diskriminierungsrichtlinienvorschlag und Gesetzgebungstätigkeit zum Lohngefälle; Konsequenzen aus dem Urteil des Bundesverfassungsgerichts zum Lissabon-Vertrag für künftige Antidiskriminierungspolitik, Justiz, Inneres und Migration; Chancengleichheit in der erwarteten „Neuauflage“ der Lissabon-Strategie; Gleichstellungsfragen bei der justiziellen $\mathrm{Zu}$ sammenarbeit; Entwicklung der Migration im Rahmen der Arbeitnehmerfreizügigkeit der neuen EU-Staaten ab 2012; Rechte türkischer Staatsangehöriger vor dem Hintergrund der aktuellen EuGH-Rechtsprechung; EU-Migrationspolitik (Frontex, Sicherheitstechnik, Grenzschutz). Öffentliches Recht: AGG im Öffentlichen Dienstrecht. Migrationsrecht: Evaluation des Nationalen Integrationsplans und des sogenannten Integrationsmonitorings; Entwicklung bei der Anerkennung von Bildungs- und Berufsabschlüssen; Evaluation des Arbeitsmigrationsgesetzes und des Gesetzes zur Umsetzung aufenthaltsund asylrechtlicher Richtlinien der Europäischen Union; irreguläre Migration; Frauen mit Migrationshintergrund im Alter.

\section{Der djb gratuliert}

\section{Prof. Dr. Dr. h.c. mult. Jutta Limbach erhält Savigny-Medaille}

Die älteste Juristische Gesellschaft Deutschlands feierte am 9. Mai 2009 mit einem Festakt in der Berlin-Brandenburgischen Akademie der Wissenschaften ihr 150-jähriges Bestehen. 1859 auf Initiative eines Redakteurs der „Preußischen Gerichtszeitung " von 14 Juristen als wissenschaftlicher Verein in Berlin gegründet, sah sie ihre Aufgabe damals wie heute darin, die Praktiker mit neuen Entwicklungen des Rechtslebens vertraut zu machen und den Rechtslehrern diese Erfahrungen mitzuteilen, um zur Verbreitung einer wissenschaftlichen Behandlung des Rechts beizutragen. Bereits 1860 gab sie den Anstoß zur Gründung des Deutschen Juristentages. Von 1859 bis zum erzwungenen vorläufigen Ende ihres Wirkens 1933 beobachtete, kritisierte und stimulierte die in der Juristischen Gesellschaft versammelte Juristenelite die deutsche Rechtsentwicklung, wie die Reihe ihrer Mitglieder und Vortragenden aus dem In- und Ausland sowie das große Themenspektrum belegen. 1959 nahm die Gesellschaft ihre Tätigkeit mit monatlichen Vorträgen, Symposien und Podiumsdiskussionen wieder auf und begründete im Verlag de Gruyter eine Schriftenreihe mit ausgewählten Beiträgen. Mit der Stiftung der Savigny-Medaille im Jahr 1986 knüpfte die Juristische Gesellschaft an die 1861 von ihr begründete Savigny-Stiftung an. Für „herausragende Verdienste um die Rechtswissenschaft“ ehrte die Gesellschaft in ihrer Festveranstaltung die frühere Präsidentin des
Bundesverfassungsgerichts, Frau Professorin Dr. Dr. h.c. mult. Jutta Limbach. Informationen über die Gesellschaft unter www.juristische-gesellschaft.de.

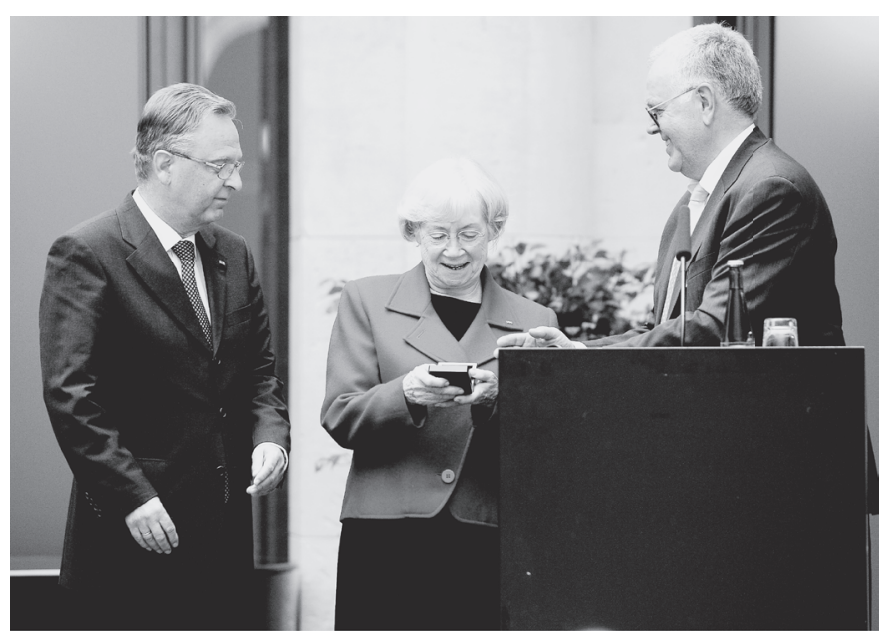

A Prof. Dr. Klaus Geppert, Präsident der Juristischen Gesellschaft zu Berlin, überreicht Prof. Dr. Dr. h.c. mult. Jutta Limbach, von 2002 bis 2008 Präsidentin des Bundesverfassungsgerichts, in Gegenwart des Präsidenten des Bundesverfassungsgerichts, Prof. Dr. Dres. h.c. Hans-Jürgen Papier, die Savigny-Medaille.

Foto: JURISTISCHE GESELLSCHAFT

\section{Jutta Wagner, Präsidentin des djb, zum 60. Geburtstag}

Liebe Jutta,

ab einem bestimmten runden Geburtstag gratuliert unsere Präsidentin den Mitgliedsfrauen mit einer freundlichen Glückwunschkarte, und da sie viele von uns persönlich kennt, fällt diese Gratulation immer individuell formuliert und persönlich aus. Das weiß ich, weil ich Jutta Wagner ab und zu vertrete. Heute ist so ein Vertretungsfall, obwohl Jutta gesund und anwesend ist, denn täte sie es selbst, läge ein rechtlich zweifelhaf- tes Insichgeschäft vor: Jutta Wagner ist 60 Jahre alt geworden, genau wie unser Verband. Das mag man nicht glauben, wenn man sie sieht, es ist aber wahr, und sie macht aus dieser erstaunlichen Zahl auch kein Hehl. Andererseits wird sie in ihrer zurückhaltenden, bescheidenen Art kein Aufhebens darum wollen und prätentiöse Gratulationen eher abwehren. Trotzdem: Solche Tage, Jubiläen, Geburtstage sind wichtig. Sie lassen uns im Strom des Alltagsgeschäftes einen Augenblick 